\title{
RAS Wild Type
}

National Cancer Institute

\section{Source}

National Cancer Institute. RAS Wild Type. NCI Thesaurus. Code C132847.

A genetic finding indicating that RAS family gene mutations are absent in a sample. 